\title{
The Temporal Logic Sugar
}

\author{
Ilan Beer ${ }^{1}$, Shoham Ben-David ${ }^{1}$, Cindy Eisner ${ }^{1}$, Dana Fisman ${ }^{1,2}$, \\ Anna Gringauze ${ }^{1}$, and Yoav Rodeh ${ }^{1,2}$ \\ 1 IBM Haifa Research Laboratory \\ 2 Weizmann Institute of Science, Rehovot, Israel \\ danaf@il.ibm.com
}

\section{Introduction}

Since the introduction of temporal logic for the specification of computer programs [5], usability has been an issue, because a difficult-to-use formalism is a barrier to the wide adoption of formal methods. Our solution is Sugar, the temporal logic used by the RuleBase formal verification tool [2]. Sugar adds the power of regular expressions to CTL [4], as well as an extensive set of operators which provide syntactic sugar. That is, while these operators do not add expressive power, they allow properties to be expressed more succinctly than in the basic language. Experience shows that Sugar allows hardware engineers to easily and intuitively specify their designs. The full language is used for model checking, and a significant portion can be model checked on-the-fly [3]. The automatic generation of simulation checkers from the same portion of Sugar is described in [1]. While previous papers have described various features of the language, this paper presents the first complete description of Sugar.

\section{The Basic Language}

We use boolean expressions to describe states in the model, and Sugar Extended Regular Expressions to describe sequences of states, and define them as follows:

\section{Definition 1. (Boolean Expression).}

1. Every atomic proposition is a boolean expression.

2. If $b, b_{1}$, and $b_{2}$ are boolean expressions, then so are $\neg b$ and $b_{1} \wedge b_{2}$.

\section{Definition 2. (Sugar Extended Regular Expressions (SEREs)).}

1. Every boolean expression is a SERE.

2. If $r, r_{1}$, and $r_{2}$ are SEREs, then so are the following: $\left.\left.i\right) r_{1}, r_{2} i i\right) r_{1} \sim r_{2}$ iii) $\left.\left.r_{1} \| r_{2} i v\right) r_{1} \& \& r_{2} v\right) r[*]$

A comma denotes concatenation, $\sim$ denotes overlapping concatenation, where the last state of $r_{1}$ coincides with the first state of $r_{2}, \|$ denotes disjunction, \&\& denotes conjunction, and $[*]$ is used to specify 0 or more repetitions.

There are two ways to use SEREs in Sugar formulas. The first is to link two SEREs in order to form Sugar formulas of the linear fragment, as defined in Definition 3 below. A second way is to link a single SERE with a general Sugar formula, as defined in Definition 4 below. 
Definition 3. (Sugar Formulas of the Linear Fragment ${ }^{1}$ ). If $r_{1}$ and $r_{2}$ are SEREs, then $\left\{r_{1}\right\} \mapsto\left\{r_{2}\right\}$ ! and $\left\{r_{1}\right\} \mapsto\left\{r_{2}\right\}$ are Sugar formulas of the linear fragment.

The $\left\{r_{1}\right\} \mapsto\left\{r_{2}\right\}$ ! and $\left\{r_{1}\right\} \mapsto\left\{r_{2}\right\}$ constructs are known as strong suffix implication and weak suffix implication, respectively. Strong suffix implications are liveness formulas, indicating that every sequence of states on which $r_{1}$ holds must be followed by a sequence of states on which $r_{2}$ holds. Weak suffix implications are safety formulas, indicating that every sequence of states on which $r_{1}$ holds may not be followed by a sequence of states which contradicts $r_{2}$. For instance, the Sugar formula $\{[*], p, q\} \mapsto\{s[*], t\}$ ! requires that every sequence of two states such that $p$ is valid in the first and $q$ is valid in the second, must be followed by a sequence of states in which $s$ is valid for some number of states, and then $t$ is valid in the final state of the sequence. The weak form of this formula does not require that the second sequence "reach its end": a sequence matching $\{p, q\}$ must be followed either by a sequence in which $s$ holds forever, or by a sequence in which $s$ holds for some number of states, and then $t$ holds.

\section{Definition 4. (Sugar Formulas).}

1. Every boolean expression is a Sugar formula.

2. Every Sugar formula of the linear fragment is a Sugar formula.

3. If $f, f_{1}$, and $f_{2}$ are Sugar formulas and $r$ is a SERE, then the following are Sugar formulas: $i) \neg f$ ii) $f_{1} \wedge f_{2}$ iii) $\left.\left.\left.E X f i v\right) E\left[f_{1} U f_{2}\right] v\right) E G f v i\right)\{r\}(f)$

The operators $\neg, \wedge, E X, E U$, and $E G$ have the usual meaning. The construct $\{r\}(f)$ (suffix implication) holds for a state $s$ if, for all finite sequences starting from $s$ on which $r$ holds, formula $f$ holds on the final state in the sequence $r$.

\section{Syntactic Sugar}

Because the basic language can be verbose, Sugar adds syntactic sugar: additional operators which allow many properties to be expressed succinctly in an intuitive manner. We will now illustrate the advantages of the syntactic sugar with a few examples.

The next_event Operators. These operators are a conceptual extension of the $A X$ operator. While $A X$ refers to the next state, next_event refers to the next state in which a boolean expression is valid. For instance, the following:

$$
A G\left(h i \_p r i \_r e q \rightarrow n e x t \_e v e n t \_f(g n t)[1 . .2]\left(d s t=h i \_p r i\right)\right)
$$

\footnotetext{
${ }^{1}$ Note that Sugar formulas of the linear fragment are not closed under the boolean operators. The result of a boolean operation on two Sugar formulas of the linear fragment is a general Sugar formula as described in Definition 4

2 The abbreviations presented here and in Appendix B are given as an explanatory semantics and do not imply the actual implementation.
} 
expresses the requirement that whenever hi_pri_req is asserted, one of the next two assertions of signal gnt must have dst equal to hi_pri.

The next_event operator, and its variant next_event_f $(b)[1 . .2](f)$ are defined in terms of the weak until $(A W)$ operator as follows: $A\left[f_{1} W f_{2}\right]$ is equivalent to $\neg E\left[\neg f_{2} U \neg f_{1} \wedge \neg f_{2}\right]$, next_event $(b)(f)$ is equivalent to $A[\neg b W b \wedge f]$, and next_event $(b)[1 . .2](f)$ is equivalent to next_event $(b)(f \vee A X$ next_event $(b)(f))$. Thus, Formula 1 can be expressed in CTL with the addition of the $A W$ operator as follows:

$$
\begin{gathered}
A G\left(h i _ { - } p r i \_ r e q \rightarrow A \left[\neg g n t W \left(\left(g n t \wedge d s t=h i_{-} p r i\right) \vee\right.\right.\right. \\
\left.\left.\left.\left(g n t \wedge A X A\left[\neg g n t W\left(g n t \wedge d s t=h i_{-} p r i\right)\right]\right)\right)\right]\right)
\end{gathered}
$$

The within Operators. The within operators ease the expression of requirements such as the following: "every transaction must complete, and within every transaction, a full data transfer must occur", which is expressible in Sugar as:

$$
A G \text { within! }\left(t r \_s t r t, t r \_e n d\right)\left\{\text { true }[*], d a t \_s t r t, \text { true }[*], d a t \_e n d\right\}
$$

within! $\left(r_{1}, b\right)\left\{r_{2}\right\}$ is equivalent to $\left\{r_{1}\right\} \mapsto\left\{r_{2} \& \&\{\neg b[*]\}, \neg b[*], b\right\}$ !. Thus, Formula 3 can be expressed (albeit somewhat cryptically) in CTL as follows:

$$
\begin{aligned}
& A G\left(t r _ { - } s t r t \rightarrow A \left[\neg d a t_{-} s t r t \wedge \neg t r_{-} \text {end } U\right.\right. \\
& \text { dat_strt } \left.\left.\wedge A\left[\neg t r_{-} \text {end } U \text { dat_end } \wedge A\left[\neg t r_{-} \text {end } U \text { tr_end }\right]\right]\right]\right)
\end{aligned}
$$

Counters. Counters are used to describe sequences of events that would otherwise be tedious to specify. For example, $i$ consecutive occurrences of sequence $r$ can be expressed as $r[i]$, and $i$ non-consecutive occurrences of boolean expression $b$ can be expressed as $b[=i]$. Formally, $r[0]$ is equivalent to false $[*]$, while $r[i]$ is equivalent to $i$ concatenations of $r$, and $b[=i]$ is equivalent to $\{\neg b[*], b\}[i], \neg b[*]$. The utility of the $b[=i]$ construct is illustrated in the following Sugar formula:

$$
A G(\{g o,\{\operatorname{get}[=8]\} \& \&\{\operatorname{kill}[=0]\}\} \mapsto\{\text { true, }\{p u t[=8]\} \& \&\{\operatorname{end}[=0]\}\})
$$

which expresses the requirement that a sequence beginning with the assertion of signal go, and containing eight not necessarily consecutive assertions of signal get, during which signal kill is not asserted, must be followed by a sequence containing eight assertions of signal put before signal end can be asserted. The equivalent CTL formula is both non-intuitive and tedious. The CTL formula expressing the same requirement but for sequences of only two gets and puts illustrates this point:

$$
\begin{aligned}
& A G \neg(\text { go } \wedge E X \quad E[\neg \text { get } \wedge \neg \text { kill } U \text { get } \wedge \neg \text { kill } \wedge \text { EX } E[\neg \text { get } \wedge \neg \text { kill } U \text { get } \wedge \neg \text { kill } \\
& \wedge E[\neg \text { put } U \text { end }] \vee E[\neg \text { put } \wedge \neg \text { end } U(\text { put } \wedge \neg \text { end } \wedge E X E[\neg \text { put } U \text { end }])]])(6)
\end{aligned}
$$

Formulas 1 [3] and [5]can also be expressed in LTL [6]. However, the equivalent LTL formulas are not any less daunting to code or decipher than the CTL versions, while the Sugar version expresses the requirements succinctly, in a manner accessible to the non-logician. 


\section{References}

1. Y. Abarbanel and I. Beer et al. FoCs - automatic generation of simulation checkers from formal specifications. In $C A V$ '00, LNCS 1855. Springer-Verlag, 2000.

2. I. Beer, S. Ben-David, C. Eisner, and A. Landver. RuleBase: an industry-oriented formal verification tool. In $D A C$ '96, pages 655-660, June 1996.

3. I. Beer, S. Ben-David, and A. Landver. On-the-fly model checking of RCTL formulas. In $C A V$ '98, LNCS 1427, pages 184-194. Springer-Verlag, 1998.

4. E. Clarke and E. Emerson. Design and synthesis of synchronization skeletons using branching time temporal logic. In Proc. Workshop on Logics of Programs, LNCS 131, pages 52-71. Springer-Verlag, 1981.

5. A. Pnueli. The temporal logic of programs. In Proc. $18^{\text {th }}$ Annual IEEE Symposium on Foundations of Computer Science, pages 46-57, 1977.

6. A. Pnueli. A temporal logic of concurrent programs. Theoretical Computer Science, 13:45-60, 1981.

\section{A Formal Semantics}

The semantics of a Sugar formula are defined with respect to a model $M$. A model is a quintuple $\left(S, S_{0}, R, P, L\right)$, where $S$ is a finite set of states, $S_{0} \subseteq S$ is a set of initial states, $R \subseteq S \times S$ is the transition relation, $P$ is a non-empty set of atomic propositions, and $L$ is the valuation, a function $L: S \longrightarrow 2^{P}$, mapping each state with a set of atomic propositions true in that state. $R$ is total with respect to its first argument. A computation path $\pi$ of a model $M$ is an infinite sequence of states $\pi=\left(\pi_{0}, \pi_{1}, \pi_{2}, \cdots\right)$ such that $R\left(\pi_{i}, \pi_{i+1}\right)$ for every $i$. We will denote by $\pi^{i, j}$ a finite sequence of states starting from $\pi_{i}$ and ending in $\pi_{j}$.

The semantics of SEREs are defined over the alphabet $2^{P}$. Thus, a letter is a subset of the set of atomic propositions $P$. We will denote a letter from $2^{P}$ by $\ell$ and a finite word over $2^{P}$ by $w$. The concatenation of $w_{1}$ and $w_{2}$ is denoted by $w_{1} w_{2}$. The empty word is denoted by $\epsilon$, so that $w \epsilon=\epsilon w=w$. The notation $w \in \mathcal{L}(r)$, where $r$ is a SERE, means that $w$ is in the language of $r$. The semantics of SEREs are defined as follows:

1. $w \in \mathcal{L}(p) \Longleftrightarrow$ there exists an $\ell$ s.t. $w=\ell$ and $p \in \ell$

2. $w \in \mathcal{L}(\neg b) \Longleftrightarrow w \notin \mathcal{L}(b)$

3. $w \in \mathcal{L}\left(b_{1} \wedge \overleftrightarrow{\left.b_{2}\right)} \Longleftrightarrow w \in \mathcal{L}\left(b_{1}\right)\right.$ and $w \in \mathcal{L}\left(b_{2}\right)$

4. $w \in \mathcal{L}\left(r_{1}, r_{2}\right) \Longleftrightarrow$ there exist $w_{1}$ and $w_{2}$ s.t. $w=w_{1} w_{2}$ and $w_{1} \in \mathcal{L}\left(r_{1}\right)$ and $w_{2} \in \mathcal{L}\left(r_{2}\right)$

5. $w \in \mathcal{L}\left(r_{1} \sim r_{2}\right) \Longleftrightarrow$ there exist $w_{1}, w_{2}$, and $\ell$ s.t. $w=w_{1} \ell w_{2}$ and $w_{1} \ell \in \mathcal{L}\left(r_{1}\right)$ and $\ell w_{2} \in \mathcal{L}\left(r_{2}\right)$

6. $w \in \mathcal{L}\left(r_{1}|| r_{2}\right) \Longleftrightarrow w \in \mathcal{L}\left(r_{1}\right)$ or $w \in \mathcal{L}\left(r_{2}\right)$

7. $w \in \mathcal{L}\left(r_{1} \& \& r_{2}\right) \Longleftrightarrow w \in \mathcal{L}\left(r_{1}\right)$ and $w \in \mathcal{L}\left(r_{2}\right)$

8. $w \in \mathcal{L}(r[*]) \Longleftrightarrow$ either $w=\epsilon$ or there exist $w_{1}, w_{2}, \ldots, w_{j}$ s.t. $w=w_{1} w_{2} \ldots w_{j}$ and, for all $i$, $1 \leq i \leq j, w_{i} \in \mathcal{L}(r)$

Recall that every state $s \in S$ in a model $M=\left(S, S_{0}, R, P, L\right)$ is associated with a set of atomic propositions by the valuation $L$. We define $\hat{L}$, an extension of the valuation function $L$ as follows: $\hat{L}\left(\pi_{i}, \pi_{i+1}, \ldots \pi_{j}\right)=L\left(\pi_{i}\right) L\left(\pi_{i+1}\right) \ldots L\left(\pi_{j}\right)$. Thus we have a mapping from states in $\mathrm{M}$ to letters of $2^{P}$, and from finite sequences of states in $\mathrm{M}$ to words over $2^{P}$.

We now turn to the semantics of Sugar formulas. The notation $M, s=f$ means that formula $f$ holds in state $s$ of model $M$. The notation $M \models f$ is equivalent to $\forall s \in S_{0} M, s \models f$, in other words, $f$ is valid for all initial states of $M$. We use $p, p_{1}$ and $p_{2}$ to denote atomic propositions, $b$, $b_{1}$ and $b_{2}$ to denote boolean expressions, $r, r_{1}$ and $r_{2}$ to denote SEREs, and $f, f_{1}$ and $f_{2}$ to denote Sugar formulas. The semantics of a Sugar formula are defined as follows:

1. $M, s=p \Longleftrightarrow p \in L(s)$

2. $M, s=\neg \Longleftrightarrow p \Leftrightarrow M, s \mid \forall f$

3. $M, s=f_{1} \wedge f_{2} \Longleftrightarrow M, s \models f_{1}$ and $M, s \models f_{2}$

4. $M, s=r_{1} \mapsto r_{2}$ ! $\Longleftrightarrow$ for all paths $\pi$ s.t. $\pi_{0}=s$, for all $j$ s.t. $\hat{L}\left(\pi^{0, j}\right) \in \mathcal{L}\left(r_{1}\right)$, there exists a $k$ s.t. $\hat{L}\left(\pi^{j, k}\right) \in \mathcal{L}\left(r_{2}\right)$

5. $M, s \models r_{1} \mapsto r_{2} \Longleftrightarrow$ for all paths $\pi$ s.t. $\pi_{0}=s$, for all $j$ s.t. $\hat{L}\left(\pi^{0, j}\right) \in \mathcal{L}\left(r_{1}\right)$, either there exists a $k$ s.t. $\hat{L}\left(\pi^{j, k}\right) \in \mathcal{L}\left(r_{2}\right)$, or for all $k$, there exists a word $w$ (not necessarily a computation path in $M)$ s.t. $\hat{L}\left(\pi^{j, k}\right) w \in \mathcal{L}\left(r_{2}\right)$

6. $M, s=E X f \Longleftrightarrow$ for some path $\pi$ s.t. $\pi_{0}=s, M, \pi_{1}=f$

7. $M, s=E\left[f_{1} U f_{2}\right] \Longleftrightarrow$ for some path $\pi$ s.t. $\pi_{0}=s$, there exists $k$ s.t. $M, \pi_{k} \models f_{2}$ and for all $j$ s.t. $j<k, M, \pi_{j}=f_{1}$

8. $M, s \models E G f \Longleftrightarrow$ for some path $\pi$ s.t. $\pi_{0}=s$, for all $j \geq 0, M, \pi_{j} \models f$

9. $M, s=\{r\}(f) \Longleftrightarrow$ for all paths $\pi$ s.t. $\pi_{0}=s$, for all $j$ s.t. $\hat{L}\left(\pi^{0, j}\right) \in \mathcal{L}(r), M, \pi_{j} \models f$ 


\section{B The Full Syntactic Sugar}

Additional boolean operators
1. $b_{1} \vee b_{2}=\neg\left(\neg b_{1} \wedge \neg b_{2}\right)$
2. $b_{1} \rightarrow b_{2}=\neg b_{1} \vee b_{2}$
2. $b_{1} \rightarrow b_{2}=\neg b_{1} \vee b_{2}$
4. $b_{1} \oplus b_{2}=\left(b_{1} \wedge \neg b_{2}\right) \vee\left(\neg b_{1} \wedge b_{2}\right)$

Additional SERE operators (where $i, j$, and $k$ are integer constants s.t. $i \geq 0, j \geq i, k>0$ )

1. $r[+]=r, r[*] \quad$ 2. $\quad r[i]=\left\{\begin{array}{lll}\begin{array}{l}\text { false }[*] \\ i \text { times }\end{array} & \text { if } i=0 & \text { 3. } r[i . . j]=r[i]\|r[i+1]\| \ldots \| r[j] \\ \overbrace{r, r, \ldots, r} & \text { otherwise }\end{array}\right.$

4. $r[i .]=.r[i], r[*]$ 5. $\quad r[. . i]=r[0]\||| r[1]\| r[2]\|\ldots\| r[i] \quad 6 . \quad[+]=\operatorname{true}[+]$

7. $[*]=\operatorname{true}[*] \quad 8 . \quad[i]=\operatorname{true}[i] \quad 9 . \quad[i . . j]=\operatorname{true}[i . . j]$

10. $[i .]=.\operatorname{true}[i .] \quad 11 ..[. . i]=\operatorname{true}[. . i] \quad 12 . b[=i]=\{\neg b[*], b\}[i], \neg b[*]$

13. $b[>i]=b[=i+1],[*] 14 . b[<k]=b[=0]\|b[=1]\| \ldots \| b[=(k-1)]$

15. $b[\geq i]=b[>i]|| b[=i] \quad 16 . b[\leq i]=b[<i] \| b[=i] \quad 17 . b[>i,<j]=b[>i] \& \& b[<j]$

18. $b[\geq i,<j]=b[\geq i] \& \& b[<j] 19 . b[>i, \leq j]=b[>i] \& \& b[\leq j] 20 . b[\geq i, \leq j]=b[\geq i] \& \& b[\leq j]$

Additional linear operators

1. always $\{r\}=\{\operatorname{true}[*]\} \mapsto\{r\} \quad$ 2. never $\{r\}=\{\operatorname{true}[*], r\} \mapsto \quad\{$ false $\}$

3. eventually $\{r\}=\{$ true $\} \mapsto\{$ true $[*], r\} ! 4$. within! $\left(r_{1}, b\right)\left\{r_{2}\right\}=\left\{r_{1}\right\} \mapsto\left\{r_{2} \& \& b[=0], \neg b[*], b\right\}$ !

5. $\operatorname{within}\left(r_{1}, b\right)\left\{r_{2}\right\}=\left\{r_{1}\right\} \mapsto\left\{r_{2} \& \& b[=0]\right\}$

6. within!_( $\left.r_{1}, b\right)\left\{r_{2}\right\}=\left\{r_{1}\right\} \mapsto\left\{\left\{r_{2} \& \& b[=0], \neg b[*], b\right\} \|\left\{r_{2} \& \&\{\neg b[*], b\}\right\}\right\}$ !

7. within_ $_{\mathbf{A}}\left(r_{1}, b\right)\left\{r_{2}\right\}=\left\{r_{1}\right\} \mapsto\left\{r_{2} \& \&\{\neg b[*]\right.$, true $\left.\}\right\}$ 8.whilenot! $(b)\{r\}=$ within!(true, $\left.b\right)\{r\}$

9. whilenot $(b)\{r\}=$ within (true, $b)\{r\} \quad$ 10. whilenot!_ $(b)\{r\}=$ within!_(true, $b)\{r\}$

11. whilenot_(b) $\{r\}=$ within_(true,$b)\{r\}$ wh $_{-}\left\{r_{1}\right\} \Leftrightarrow\left\{r_{2}\right\} !=\left\{r_{1}\right\} \mapsto\left\{\right.$ true, $\left.r_{2}\right\}$ !

13. $\left\{r_{1}\right\} \mapsto\left\{r_{2}\right\}=\left\{r_{1}\right\} \mapsto$ true, $\left.r_{2}\right\}$

Additional branching operators (where $i$ and $j$ are integers s.t. $i>0$ and $j \geq i$ )

$\begin{array}{lll}\text { 1. } E F f=E[\operatorname{true} U f] & \text { 2. } A X f=\neg E X \neg f & 3 G f=\neg E[\text { true } U \neg f]\end{array}$

4. $A\left[f_{1} U f_{2}\right]=\neg\left(E\left[\neg f_{2} U \neg f_{1} \wedge \neg f_{2}\right] \vee E G \neg f_{2}\right)$

5. $A F f=A[$ true $U f]$ 6. $E\left[\begin{array}{lll}f_{1} & W & f_{2}\end{array}\right]=E\left[f_{1} U f_{2}\right] \vee E G f_{1} 7 . A\left[f_{1} W f_{2}\right]=\neg E\left[\neg f_{2} U \neg f_{1} \wedge \neg f_{2}\right]$

8. $A X[i] f=\overbrace{A X A X \ldots A X}^{\text {i times }} f \quad$ 9. $A B G[i . . j] f=A X[i] f \wedge A X[i+1] f \wedge \ldots \wedge A X[j] f$

10. $A B F[i . . j] f=A X[i](\overbrace{f \vee A X(f \vee A X(\ldots f \vee A X}(f) \ldots)))$

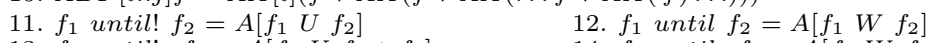

13. $f_{1}$ until! $f_{-}=A\left[f_{1} U f_{1} \wedge f_{2}\right]$

15. $f_{1}$ before! $f_{2}=A\left[\neg f_{2} U f_{1}\right]$

17. $f_{1}$ before! - $f_{2}=A\left[\neg f_{2} U f_{1} \wedge \neg f_{2}\right]$

19. next_event! $(b)(f)=A[\neg b U b \wedge f]$

21. next_event $!(b)[i](f)=\underset{i-1 \text { times }}{\text { next_event } !(b)}$

14. $f_{1}$ until $-f_{2}=A\left[f_{1} W f_{1} \wedge f_{2}\right]$

16. $f_{1}$ before $f_{2}=A\left[\neg f_{2} W f_{1}\right]$

18. $f_{1}$ before $f_{2}=A\left[\neg f_{2} W f_{1} \wedge \neg f_{2}\right]$

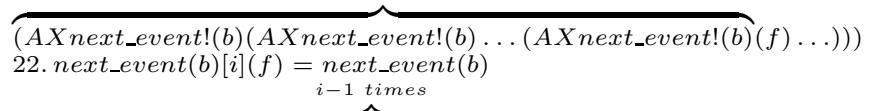

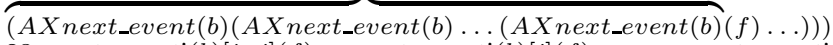

23. next_event! $(b)[i . . j](f)=$ next_event $(b)[i](f) \wedge \ldots \wedge$ next_event $!(b)[j](f)$

24. next_event $(b)[i . . j](f)=$ next_event $(b)[i](f) \wedge \ldots \wedge$ next_event $(b)[j](f)$

25. next_event_f! $(b)[i . . j](f)=$ next_event! $(b)[i]$

$j-i$ times

$\overbrace{\left(f \vee A X n e x t \_e v e n t !(b)\left(f \vee A X n e x t \_e v e n t !(b) \ldots\left(f \vee A X n e x t \_e v e n t !(b)\right.\right.\right.}(f) \ldots)))$

26. next_event_f $(b)[i . . j](f)=$ next_event $(b)[i]$ $j-i$ times

$\overbrace{\left(f \vee A X n e x t_{-} \text {event }(b)\left(f \vee A X n e x t \_ \text {event }(b) \ldots\left(f \vee A X n \operatorname{ext} \_ \text {event }(b)\right.\right.\right.}(f) \ldots)))$ 